CUBO A Mathematical Journal Vol.15, № 01, (13-32). March 2013

\title{
Solow Models on Time Scales 1
}

\author{
Martin Bohner And Julius Heim \\ Missouri University of Science and Technology, \\ Department of Mathematics and Statistics, \\ Department of Economics, \\ Rolla, MO 65409-0020, USA \\ bohner@mst.edu \\ julius.heim@mst.edu
}

\author{
Ailian LiU \\ Shandong University of Finance and \\ Economics, \\ School of Mathematics and Quantitative \\ Economics, \\ Jinan 250014, P. R. China \\ ailianliu20020163. com
}

\begin{abstract}
We introduce a general Solow model on time scales and derive a nonlinear first-order dynamic equation that describes such a model. We first assume that there is neither technological development nor a change in the population. We present the CobbDouglas production function on time scales and use it to give the solution for the equation that describes the model. Next, we provide several applications of the generalized Solow model. Finally, we generalize our work by allowing technological development and population growth. The presented results not only unify the continuous and the discrete Solow models but also extend them to other cases "in between", e.g., a quantum calculus version of the Solow model. Finally it is also noted that our results even generalize the classical continuous and discrete Solow models since we allow the savings rate, the depreciation factor of goods, the growth rate of the population, and the technological growth rates to be functions of time rather than taking constant values as in the classical Solow models.
\end{abstract}

Keywords and Phrases: Time scales, Solow model, dynamic equation, Cobb-Douglas production function, economics.

2010 AMS Mathematics Subject Classification: 91B64, 34C10, 39A10, 39A11, 39A12, $39 \mathrm{~A} 13$.

\footnotetext{
${ }^{1}$ This paper is dedicated to Professor Gaston M. N'Guérékata on the occasion of his 60th birthday
} 


\section{RESUMEN}

Introducimos un modelo general de Solow en escalas de tiempo y derivamos una ecuación dinámica no lineal de primer orden que describe el mencionado modelo. Primero asumimos que no existe ni desarrollo tecnológico ni un cambio en la población. Presentamos la función de producción de Cobb-Douglas en escalas de tiempo y la utilizamos para entregar la solución de la ecuación que describe el modelo. Luego, mostramos varias aplicaciones del modelo generalizado de Solow. Finalmente, generalizamos nuestro trabajo permitiendo desarrollo tecnológico y crecimiento de la población. Los resultados presentados no sólo unifican los modelos de Solow continuos y discretos, sino que además se extienden a otros casos "entre medio", es decir, una versión del cálculo cuántico del modelo de Solow. Finalmente, también se menciona que nuestro resultado también generaliza los modelos clásicos continuos y discretos, ya que permitimos tasas de ahorro, el factor de depreciación de bienes, la razón de crecimiento de la población y las razones de crecimiento tecnológico por ser funciones del tiempo más que asumiendo valores constantes como es el caso de los modelos de Solow clásico.

\section{The Classical Solow Model}

Modern growth theory is mainly based on the works of Solow [12] and Swan [13]. In the Solow model, it is assumed that the national income $\mathrm{Y}$ depends on consumption $\mathrm{C}$ and investment I, i.e.,

$$
Y(t)=C(t)+I(t)
$$

Moreover, it is assumed that the national income is a function of the capital stock $\mathrm{K}$ and the product of the technological progress $\mathrm{A}$ and the population $\mathrm{N}$, i.e.,

$$
\mathrm{Y}(\mathrm{t})=\mathrm{F}(\mathrm{K}(\mathrm{t}), \mathrm{A}(\mathrm{t}) \mathrm{N}(\mathrm{t}))
$$

where the production function $F$ satisfies the following conditions:

1. $F(\lambda K, \lambda L)=\lambda F(K, L)$ for all $\lambda, K, L \in \mathbb{R}^{+}$(constant returns to scale);

2. $\mathrm{F}(\mathrm{K}, \mathrm{O})=\mathrm{F}(\mathrm{O}, \mathrm{L})=0$ for all $\mathrm{K}, \mathrm{L} \in \mathbb{R}^{+}$;

3. $\frac{\partial F}{\partial K}>0, \frac{\partial F}{\partial L}>0, \frac{\partial^{2} F}{\partial K^{2}}<0, \frac{\partial^{2} F}{\partial L^{2}}<0$;

4. $\lim _{\mathrm{K} \rightarrow 0^{+}} \frac{\partial \mathrm{F}}{\partial \mathrm{K}}=\lim _{\mathrm{L} \rightarrow 0^{+}} \frac{\partial \mathrm{F}}{\partial \mathrm{L}}=+\infty, \lim _{\mathrm{K} \rightarrow+\infty} \frac{\partial \mathrm{F}}{\partial \mathrm{K}}=\lim _{\mathrm{L} \rightarrow+\infty} \frac{\partial \mathrm{F}}{\partial \mathrm{L}}=0$.

Furthermore, the change of the capital stock in a particular period does not only depend on the new investment but also on the depreciation of goods. In other words, we take for granted that

$$
K^{\prime}(t)=I(t)-\delta K(t)
$$


with given initial capital stock $\mathrm{K}(0)$, where $\delta$ is the depreciation rate of the goods. As usual, we presume that

$$
S(t)=I(t)=s Y(t),
$$

where $s$ is the savings rate, and thus the savings $S$ is the portion of the national income which is not consumed. Plugging (11) and (3) into (2), we obtain the nonlinear first-order differential equation

$$
K^{\prime}(t)=s F(K(t), A(t) N(t))-\delta K(t) .
$$

In this paper, we assume that the technological knowledge of the society grows exponentially with rate $r$, that is,

$$
A^{\prime}(t)=r A(t), \quad \text { i.e., } \quad A(t)=e^{r t} A(0),
$$

where $A(0)$ is the initial technological standing of the nation. Similarly, we suppose that the population of the nation grows with growth rate $n$ and an initial population of $N(0)$, hence

$$
\mathrm{N}^{\prime}(\mathrm{t})=\mathrm{nN}(\mathrm{t}), \quad \text { i.e., } \quad \mathrm{N}(\mathrm{t})=\mathrm{e}^{\mathrm{nt}} \mathrm{N}(0) \text {. }
$$

Both $n$ and $r$ can be positive or negative depending on which nation we are talking about. Of course, it seems quite unrealistic that $r$ is negative. Solow also took the capital stock per efficiency of labor $\mathrm{k}$ into account, which he defined as the stationary variable

$$
k(t):=\frac{K(t)}{A(t) N(t)}
$$

We use constant returns in order to define the intensive version of the production function to be

$$
y(t):=\frac{Y(t)}{A(t) N(t)}=\frac{F(K(t), A(t) N(t))}{A(t) N(t)}=F(k(t), 1)=: f(k(t)) .
$$

This allows us now to rewrite (4) as

$$
\frac{K^{\prime}(t)}{A(t) N(t)}=s f(k(t))-\delta k(t)
$$

A simple calculation shows that

$$
\frac{k^{\prime}(t)}{k(t)}=\frac{K^{\prime}(t)}{K(t)}-r-n
$$

Therefore (5) turns into

$$
k^{\prime}(t)=s f(k(t))-(\delta+n+r) k(t) .
$$

Table 1 summarizes all the variables with their meanings that appear in the Solow model. For a more detailed discussion of stability and qualitative analysis of the Solow model, the reader might consult [9. The discrete analogue of Solow's model is discussed in [11, featuring some results similar to those in the continuous Solow model. So far this dynamic process was regarded either as solely continuous or solely discrete. In this paper, we generalize these two theories in such a way that the continuous and the discrete versions of the Solow model are only special cases of 
Table 1: Explanation of Variables

\begin{tabular}{|c||c|}
\hline Variable & Explanation \\
\hline \hline Y & national income \\
\hline I & induced investment \\
\hline C & consumption \\
\hline s & Savings \\
\hline K & cavings rate \\
\hline$K_{0}$ & initial capital stock \\
\hline$\delta$ & level of technological knowledge \\
\hline$A$ & initial technological progress level \\
\hline$A_{0}$ & technological growth rate \\
\hline$r$ & level of population \\
\hline$N$ & initial population \\
\hline$N_{0}$ & growth rate of population \\
\hline$n$ & capital stock per efficiency unit of labor \\
\hline $\mathrm{k}$ & \\
\hline
\end{tabular}

our generalized Solow model. We do this using the time scales theory, an area of mathematics which was originally introduced by Stefan Hilger in his $\mathrm{PhD}$ thesis [10]. The two books [7, 8] by Bohner and Peterson offer an introduction with applications to time scales calculus along with some advanced topics. Applications of time scales calculus can be found in many areas, also in economics. Tisdell and Zaidi [14] in particular already generalized some economic topics, and also Bohner et al. discussed multiplier-accelerator models and utility functions on time scales in [5, 6].

The set up of this paper is as follows. In Section 2, we give a brief introduction to the time scales theory. In Section 3, we present the Solow model on time scales and derive the nonlinear first-order dynamic equation that describes this model. In Section 4, we define the generalized Cobb-Douglas production function on time scales and provide examples for various time scales. Furthermore, we state a theorem that gives the solution of the nonlinear first-order dynamic equation, and we also provide examples for several time scales. We finally state a result that addresses asymptotic stability of the solution. Section 5 is used to show some important properties of the production function, called the Inada conditions. Finally, in Section 6, we point out how our model can be extended, assuming the presence of technological and population growth (or decay), i.e., by assuming $n \neq 0$ as well as $r \neq 0$. We present the Cobb-Douglas production function for 
this more general case and also derive the equilibrium solution for this extended model.

It is also noted that our results even generalize the classical continuous and discrete Solow models since we allow the savings rate, the depreciation factor of goods, the growth rate of the population, and the technological growth rates to be functions of time rather than considering constant values as in the classical Solow models.

\section{Time Scales Preliminaries}

In this section, we introduce some elements of time scales calculus. For a more rigorous time scales introduction, we refer the reader to 7,8 .

Let $\mathbb{T}$ be a time scale, i.e., a nonempty closed subset of $\mathbb{R}$. For $t \in \mathbb{T}$, the forward jump operator $\sigma: \mathbb{T} \rightarrow \mathbb{T}$ is defined by

$$
\sigma(t):=\inf \{s \in \mathbb{T}: s>t\}
$$

while the backward jump operator $\rho: \mathbb{T} \rightarrow \mathbb{T}$ is defined by

$$
\rho(t):=\sup \{s \in \mathbb{T}: s<t\} .
$$

In this definition, we set $\inf \emptyset=\sup \mathbb{T}$ (i.e., $\sigma(t)=t$ if $\mathbb{T}$ has maximum $t$ ) and $\sup \emptyset=\inf \mathbb{T}$ (i.e., $\rho(t)=t$ if $\mathbb{T}$ has minimum $t$ ). If $\sigma(t)>t, \sigma(t)=t, \rho(t)<t$, and $\rho(t)=t$, then $t$ is called right-scattered, right-dense, left-scattered and left-dense, respectively. The graininess function $\mu$ : $\mathbb{T} \rightarrow[0, \infty)$ is defined by

$$
\mu(t):=\sigma(t)-t
$$

We also need the set $\mathbb{T}^{\kappa}$ which is defined in the following way: If $\mathbb{T}$ has a left-scattered maximum $\mathrm{m}$, then $\mathbb{T}^{\mathrm{k}}=\mathbb{T}-\{\mathrm{m}\}$. Else, $\mathbb{T}^{\mathrm{K}}=\mathbb{T}$.

Now let $f: \mathbb{T} \rightarrow \mathbb{R}$ be a function. If $t \in \mathbb{T}^{\kappa}$, then $f^{\Delta}(t)$ is defined as the number (provided that it exists) such that for every $\varepsilon>0$, there exists a neighborhood $\mathrm{U}$ of $\mathrm{t}$ (i.e., $\mathrm{U}=(\mathrm{t}-\delta, t+\delta) \cap \mathbb{T}$ for some $\delta>0$ ) such that

$$
\left|[f(\sigma(t))-f(s)]-f^{\Delta}(t)[\sigma(t)-s]\right| \leq \varepsilon|\sigma(t)-s| \quad \text { for all } \quad s \in U
$$

We call this number $\mathrm{f}^{\Delta}(\mathrm{t})$ the delta derivative of $\mathrm{f}$ at $\mathrm{t}$. Moreover, $\mathrm{f}$ is called $r d$-continuous provided it is continuous at right-dense points in $\mathbb{T}$ and its left sided limits exist (finite) at left-dense points in $\mathbb{T}$. The function $f^{\sigma}: \mathbb{T} \rightarrow \mathbb{R}$ is defined by $f^{\sigma}=f \circ \sigma$. In our calculations, we use the so-called "simple useful formula"

$$
f^{\sigma}=f+\mu f^{\Delta}
$$

We denote the set of rd-continuous functions by $\mathrm{C}_{\mathrm{rd}}=\mathrm{C}_{\mathrm{rd}}(\mathbb{T})=\mathrm{C}_{\mathrm{rd}}(\mathbb{T}, \mathbb{R})$. Next, $\mathrm{f}$ is said to be regressive given that

$$
1+\mu(t) f(t) \neq 0 \quad \text { for all } t \in \mathbb{T}
$$


holds. The set of all regressive and rd-continuous functions is denoted by $\mathcal{R}=\mathcal{R}(\mathbb{T})=\mathcal{R}(\mathbb{T}, \mathbb{R})$. We also define the set $\mathcal{R}^{+}$of all positively regressive elements by

$$
\mathcal{R}^{+}=\mathcal{R}^{+}(\mathbb{T}, \mathbb{R})=\{\mathrm{f} \in \mathcal{R}: 1+\mu(\mathrm{t}) \mathrm{p}(\mathrm{t})>0 \quad \text { for all } \mathrm{t} \in \mathbb{T}\} .
$$

Let now $p, q \in \mathcal{R}$. We define the "circle plus" addition $\oplus$ on $\mathcal{R}$ by

$$
(p \oplus q)(t):=p(t)+q(t)+\mu(t) p(t) q(t) \quad \text { for all } \quad t \in \mathbb{T}
$$

and the "circle minus" subtraction $\ominus$ on $\mathcal{R}$ by

$$
(p \ominus q)(t):=\frac{p(t)-q(t)}{1+\mu(t) q(t)} \quad \text { for all } \quad t \in \mathbb{T} .
$$

We put

$$
\mathcal{R}(\alpha):=\left\{\begin{array}{lll}
\mathcal{R} & \text { if } & \alpha \in \mathbb{N}, \\
\mathcal{R}^{+} & \text {if } & \alpha \in \mathbb{R} \backslash \mathbb{N} .
\end{array}\right.
$$

For $\alpha \in \mathbb{R}$ and $p \in \mathcal{R}(\alpha)$, we define

$$
(\alpha \odot p)(t):=\alpha p(t) \int_{0}^{1}(1+\mu(t) p(t) h)^{\alpha-1} d h .
$$

The time scales exponential function $e_{p}\left(\cdot, t_{0}\right)$ is defined for $p \in \mathcal{R}$ and $t_{0} \in \mathbb{T}$ as the unique solution of the initial value problem

$$
y^{\Delta}=p(t) y, \quad y\left(t_{0}\right)=1 \quad \text { on } \quad \mathbb{T} .
$$

We have

$$
e_{\mathfrak{p}}\left(\cdot, t_{0}\right) e_{\mathfrak{q}}\left(\cdot, t_{0}\right)=e_{p} \oplus \mathfrak{q}\left(\cdot, t_{0}\right) \quad \text { and } \quad \frac{e_{p}\left(\cdot, t_{0}\right)}{e_{\mathfrak{q}}\left(\cdot, t_{0}\right)}=e_{p \ominus q}\left(\cdot, t_{0}\right) .
$$

If $\alpha \in \mathbb{R}$ and $p \in \mathcal{R}(\alpha)$, then

$$
e_{\alpha \odot p}=e_{p}^{\alpha}
$$

(see [8, Theorem 2.44]). Let $\alpha \in \mathbb{R} \backslash\{1\}$. We say that

$$
x^{\Delta}=\left[q \ominus\left(\frac{1}{\alpha-1} \odot\left(g x^{\alpha-1}\right)\right)\right] x
$$

(see [8, Section 2.6]) is a Bernoulli equation on time scales.

\section{Solow Model on Time Scales}

Assume that $F$ and $f$ are production functions as defined in Section 1 . We now introduce the generalized Solow model on an arbitrary time scale:

$$
\begin{cases}Y(t) & =F(K(t), A(t) N(t)) \\ K^{\Delta}(t) & =I(t)-\delta(t) K(t) \\ I(t) & =s(t) Y(t) \\ A^{\Delta}(t) & =r(t) A(t) \\ N^{\Delta}(t) & =n(t) N(t)\end{cases}
$$


where we require

$$
\delta(t)>0 \text { and } s(t)>0 \text { for all } t \in \mathbb{T}
$$

and

$$
\mathrm{n}, \mathrm{r} \in \mathcal{R} \text {. }
$$

The economical meanings of $\delta, s, r$, and $n$ are the same as described in Table 1 If (K, Y, A, N, I) solves (9), then

$$
K^{\Delta}(t)=s(t) Y(t)-\delta(t) K(t)=s(t) F(K(t), A(t) N(t))-\delta(t) K(t)
$$

Define

$$
k(t):=\frac{K(t)}{A(t) N(t)} \quad \text { and } \quad y(t):=\frac{Y(t)}{A(t) N(t)}
$$

which are regarded as the capital stock per efficiency unit of labor and the production per efficiency unit of labor, respectively. By (12) and (13), we have

$$
\frac{K^{\Delta}(t)}{A(t) N(t)}=s(t) f(k(t))-\delta(t) k(t)
$$

Theorem 3.1. Assume (9), (10), and (11). If $\mathrm{k}$ is defined as in (13), then

$$
\begin{aligned}
k^{\Delta}(t)= & \frac{s(t)}{(1+\mu(t) r(t))(1+\mu(t) n(t))} f(k(t)) \\
& -\left(\frac{\delta(t)+n(t)}{(1+\mu(t) r(t))(1+\mu(t) n(t))}+\frac{r(t)}{1+\mu(t) r(t)}\right) k(t) .
\end{aligned}
$$

Proof. The time scales quotient rule [7, Theorem $1.20(\mathrm{v})$ ] provides

$$
\begin{aligned}
& k^{\Delta}=\left(\frac{K}{A N}\right)^{\Delta}=\frac{K^{\Delta} A N-K\left(A N^{\Delta}+A^{\Delta} N^{\sigma}\right)}{A N A^{\sigma} N^{\sigma}} \\
& =\frac{\mathrm{K}^{\Delta}}{\mathrm{A}^{\sigma} \mathrm{N}^{\sigma}}-\frac{\mathrm{KN}^{\Delta}}{\mathrm{N} A^{\sigma} \mathrm{N}^{\sigma}}-\frac{\mathrm{KA} A^{\Delta}}{\mathrm{AN} A^{\sigma}} \\
& \stackrel{\mathrm{K}^{\Delta}}{=} \quad \frac{\mathrm{n}}{\mathrm{AN}(1+\mu \mathrm{r})(1+\mu \mathrm{n})}-\frac{\mathrm{r}}{(1+\mu \mathrm{r})(1+\mu \mathrm{n})} \mathrm{k}-\frac{\mathrm{n}}{1+\mu \mathrm{r}} \mathrm{k} \\
& \stackrel{\text { 114 }}{=} \frac{s}{(1+\mu r)(1+\mu n)} f \circ k-\left(\frac{\delta+n}{(1+\mu r)(1+\mu n)}+\frac{r}{1+\mu r}\right) k \text {, }
\end{aligned}
$$

i.e., (15) holds.

Example 3.2. If $\mathbb{T}=\mathbb{R}$, then $\sigma(\mathrm{t})=\mathrm{t}$ and $\mu(\mathrm{t})=0$ for all $\mathrm{t} \in \mathbb{T}$. Thus (15) can be rewritten as

$$
k^{\prime}(t)=s(t) f(k(t))-(\delta(t)+n(t)+r(t)) k(t),
$$

which reduces to (6) provided $\delta, \mathrm{s}, \mathrm{n}$, and $\mathrm{r}$ are constants. 
Theorem 3.3. Assume (10) and (11). Then (15) holds if and only if

$$
k^{\Delta}(t)=s(t) f(k(t))-\delta(t) k(t)-(n \oplus r)(t) k(\sigma(t)) .
$$

Proof. Suppose k solves (16). Then we use the "simple useful formula" to obtain

$$
\begin{aligned}
k^{\Delta} & =s(f \circ k)-\delta k-(n \oplus r) k^{\sigma} \\
& =s(f \circ k)-\delta k-(n+r+\mu n r)\left(k+\mu k^{\Delta}\right) .
\end{aligned}
$$

Hence

$$
(1+\mu n)(1+\mu r) k^{\Delta}=s(f \circ k)-(\delta+n+r+\mu n r) k .
$$

Dividing by $(1+\mu n)(1+\mu r)$ yields (15). If $k$ solves (15), then (16) follows by reversing the above steps.

Theorem 3.4. Assume (10) and (11). If (16) holds, then

$$
(1+\mu(t) n(t))(1+\mu(t) r(t)) k^{\sigma}(t)=\mu(t) s(t) f(k(t))+(1-\mu(t) \delta(t)) k(t) .
$$

If (17) holds and $\mu(\mathrm{t}) \neq 0$, then (16) holds.

Proof. Suppose k solves (16). Then we multiply (16) by $\mu(\mathrm{t})$ and use the simple useful formula to obtain

$$
\begin{aligned}
k(\sigma(t))-k(t) & =\mu(t) k^{\Delta}(t) \\
& =\mu(t) s(t) f(k(t))-\mu(t) \delta(t) k(t)-\mu(t)(n \oplus r)(t) k(\sigma(t)) .
\end{aligned}
$$

Hence

$$
(1+\mu(t)(n \oplus r)(t)) k(\sigma(t))=\mu(t) s(t) f(k(t))+(1-\mu(t) \delta(t)) k(t),
$$

which results in (17). If (17) holds at $t \in \mathbb{T}$ such that $\mu(t) \neq 0$, then the above steps can be reversed.

Example 3.5. If $\mathbb{T}=\mathbb{Z}$, then $\sigma(\mathrm{t})=\mathrm{t}+1$ and $\mu(\mathrm{t})=1$ for all $\mathrm{t} \in \mathbb{T}$. Thus (17) can be rewritten as

$$
(1+n(t))(1+r(t)) k(t+1)=s(t) f(k(t))+(1-\delta(t)) k(t) .
$$

This equation can be found in [11].

Example 3.6. If $\mathbb{T}=\mathrm{h} \mathbb{Z}$ with $\mathrm{h}>0$, then $\sigma(\mathrm{t})=\mathrm{t}+\mathrm{h}$ and $\mu(\mathrm{t})=\mathrm{h}$ for all $\mathrm{t} \in \mathbb{T}$. Thus (17) can be rewritten as

$$
(1+h n(t))(1+h r(t)) k(t+h)=h s(t) f(k(t))+(1-h \delta(t)) k(t) .
$$

Example 3.7. If $\mathbb{T}=\mathrm{q}^{\mathbb{N}_{0}}$ with $\mathrm{q}>1$, then $\sigma(\mathrm{t})=\mathrm{qt}$ and $\mu(\mathrm{t})=(\mathrm{q}-1) \mathrm{t}$ for all $\mathrm{t} \in \mathbb{T}$. Thus (17) can be rewritten as

$$
(1+(q-1) \operatorname{tn}(t))(1+(q-1) \operatorname{tr}(t)) k(q t)=(q-1) \operatorname{ts}(t) f(k(t))+(1-(q-1) t \delta(t)) k(t)
$$




\section{Analysis of the Basic Solow Model}

In this section, we assume that (10) holds and that there is no technological development and no population change, i.e., $n=r=0$. Then (16) simplifies to

$$
k^{\Delta}(t)=s(t) f(k(t))-\delta(t) k(t) .
$$

Let

$$
0<\alpha<1, \quad w(t)=\left(\frac{1}{\alpha-1} \odot \frac{\delta g}{s}\right)(t), \quad \text { and } \quad g(t)=(1-\alpha) s(t)
$$

If

$$
\tilde{f}(x):=\frac{\delta(t)+\left(w \ominus\left(\frac{1}{\alpha-1} \odot\left(g x^{\alpha-1}\right)\right)\right)(t)}{s(t)} \quad \text { is independent of } t \in \mathbb{T},
$$

then we define the generalized Cobb-Douglas production function on time scales by

$$
f(x)=x \widetilde{f}(x) .
$$

Theorem 4.1. Let $\mathrm{t} \in \mathbb{T}$. If $\mu(\mathrm{t})=0$, then

$$
\frac{\delta(t)+\left(w \ominus\left(\frac{1}{\alpha-1} \odot\left(g x^{\alpha-1}\right)\right)\right)(t)}{s(t)}=x^{\alpha-1} .
$$

Proof. Assume $\mu(t)=0$. Then at $t$, we have

$$
\begin{aligned}
\frac{\delta(t)+\left(w \ominus\left(\frac{1}{\alpha-1} \odot\left(g x^{\alpha-1}\right)\right)\right)(t)}{s(t)} & =\frac{\delta(t)+w(t)-\frac{g(t) x^{\alpha-1}}{\alpha-1}}{s(t)} \\
& =\frac{\delta(t)+\frac{\delta(t) g(t)}{(\alpha-1) s(t)}-\frac{g(t) x^{\alpha-1}}{\alpha-1}}{s(t)} \\
& =\frac{\delta(t)-\delta(t)+s(t) x^{\alpha-1}}{s(t)} \\
& =x^{\alpha-1},
\end{aligned}
$$

which shows (22).

Example 4.2. If $\mathbb{T}=\mathbb{R}$, then $\widetilde{\mathrm{f}}(\mathrm{x})=x^{\alpha-1}$, and thus (20) holds. Hence the Cobb-Douglas production function is defined and equals

$$
f(x) \stackrel{(21)}{=} x \widetilde{f}(x)=x x^{\alpha-1}=x^{\alpha} .
$$

Theorem 4.3. Let $\mathrm{t} \in \mathbb{T}$. If $\mu(\mathrm{t})>0$, then

$$
\begin{aligned}
\delta(t)+\left(w \ominus\left(\frac{1}{\alpha-1} \odot\left(g x^{\alpha-1}\right)\right)\right)(t) & \\
& =\frac{1}{\mu(t)}\left\{\delta(t) \mu(t)-1+\left(\frac{1+(1-\alpha) \mu(t) s(t) x^{\alpha-1}}{1+(1-\alpha) \mu(t) \delta(t)}\right)^{\frac{1}{1-\alpha}}\right\} .
\end{aligned}
$$


Proof. Assume $\mu(t)>0$. Then at $t$, we have

$$
\begin{aligned}
\frac{1}{\alpha-1} \odot\left(g x^{\alpha-1}\right) \stackrel{7}{\equiv} \frac{1}{\alpha-1} g x^{\alpha-1} \int_{0}^{1}\left(1+\mu g x^{\alpha-1} h\right)^{\frac{1}{\alpha-1}-1} \mathrm{dh} \\
=\frac{\left(1+\mu g x^{\alpha-1}\right)^{\frac{1}{\alpha-1}}-1}{\mu}, \\
w=\frac{1}{\alpha-1} \odot \frac{\delta g}{s}=\frac{1}{\alpha-1} \odot(\delta(1-\alpha)) \\
\stackrel{\frac{7}{=}}{w} \frac{1}{\alpha-1} \delta(1-\alpha) \int_{0}^{1}(1+\mu \delta(1-\alpha) h)^{\frac{1}{\alpha-1}-1} \mathrm{dh} \\
=\frac{(1+\mu \delta(1-\alpha))^{\frac{1}{\alpha-1}}-1}{\mu}
\end{aligned}
$$

and hence

$$
\begin{aligned}
w \ominus\left(\frac{1}{\alpha-1} \odot\left(g x^{\alpha-1}\right)\right) & =\frac{w-\frac{\left(1+\mu g x^{\alpha-1}\right)^{\frac{1}{\alpha-1}}-1}{\mu}}{1+\mu \frac{\left(1+\mu g x^{\alpha-1}\right)^{\frac{1}{\alpha-1}}-1}{\mu}} \\
& =\frac{w-\frac{\left(1+\mu g x^{\alpha-1}\right)^{\frac{1}{\alpha-1}}-1}{\mu}}{\left(1+\mu g x^{\alpha-1}\right)^{\frac{1}{\alpha-1}}} \\
& =\frac{\frac{(1+\mu \delta(1-\alpha))^{\frac{1}{\alpha-1}-1}-\frac{\left(1+\mu g x^{\alpha-1}\right)^{\frac{1}{\alpha-1}}-1}{\mu}}{\left(1+\mu g x^{\alpha-1}\right)^{\frac{1}{\alpha-1}}}}{} \\
& =\frac{1}{\mu}\left\{-1+\left(\frac{1+\mu \delta(1-\alpha)}{1+\mu(1-\alpha) s x^{\alpha-1}}\right)^{\frac{1}{\alpha-1}}\right\}
\end{aligned}
$$

which shows (23).

Theorem 4.4. Let $\mu(\mathrm{t})>0$ for all $\mathrm{t} \in \mathbb{T}$. Assume (10) and suppose

$$
\widetilde{s}:=\mathrm{s}(\mathrm{t}) \mu(\mathrm{t}) \quad \text { and } \quad \widetilde{\delta}:=\delta(\mathrm{t}) \mu(\mathrm{t}) \quad \text { are independent of } \mathrm{t} \in \mathbb{T} \text {. }
$$

Then (20) holds and the Cobb-Douglas production function is defined and equals

$$
f(x)=\frac{x}{\widetilde{s}}\left\{\widetilde{\delta}-1+\left(\frac{1+(1-\alpha) \widetilde{s} x^{\alpha-1}}{1+(1-\alpha) \widetilde{\delta}}\right)^{\frac{1}{1-\alpha}}\right\}
$$


Proof. Using Proposition 4.3, we see that

$$
\begin{aligned}
& \frac{\delta(t)+\left(w \ominus\left(\frac{1}{\alpha-1} \odot\left(g x^{\alpha-1}\right)\right)\right)(t)}{s(t)} \\
& \stackrel{1233}{=} \frac{1}{\mu(t) s(t)}\left\{\delta(t) \mu(t)-1+\left(\frac{1+(1-\alpha) \mu(t) s(t) x^{\alpha-1}}{1+(1-\alpha) \mu(t) \delta(t)}\right)^{\frac{1}{1-\alpha}}\right\} \\
& =\frac{1}{\widetilde{s}}\left\{\widetilde{\delta}-1+\left(\frac{1+(1-\alpha) \widetilde{s} x^{\alpha-1}}{1+(1-\alpha) \widetilde{\delta}}\right)^{\frac{1}{1-\alpha}}\right\}
\end{aligned}
$$

is independent of $t$ and therefore equals $\widetilde{f}(x)$. By (21), $f(x)=x \widetilde{f}(x)$.

Example 4.5. If $\mathbb{T}=\mathrm{h} \mathbb{Z}$ with $\mathrm{h}>0$ and $\delta$, s are constants and satisfy (10), then Proposition 4.4 gives us that (20) is satisfied and that the Cobb-Douglas production function is defined and equals (note that (24) is satisfied in this case with $\widetilde{\mathrm{s}}=\mathrm{sh}$ and $\widetilde{\delta}=\delta \mathrm{h}$ )

$$
f(x)=\frac{x}{h s}\left\{\delta h-1+\left(\frac{1+(1-\alpha) h s x^{\alpha-1}}{1+(1-\alpha) h \delta}\right)^{\frac{1}{1-\alpha}}\right\} .
$$

Example 4.6. If $\mathbb{T}=\mathbb{Z}$ and $\delta$, s are constants and satisfy (10), then Example 4.5 gives us that (20) is satisfied and that the Cobb-Douglas production function, i.e., the discrete version of the classical Cobb-Douglas production function, is defined and equals

$$
f(x)=\frac{x}{s}\left\{\delta-1+\left(\frac{1+(1-\alpha) s x^{\alpha-1}}{1+(1-\alpha) \delta}\right)^{\frac{1}{1-\alpha}}\right\} .
$$

Example 4.7. If $\mathbb{T}=\mathrm{q}^{\mathbb{N}_{0}}$ with $\mathrm{q}>1$ and $\delta$, s satisfy (10) and (24), i.e.,

$$
\widetilde{s}:=(\mathrm{q}-1) \mathrm{ts}(\mathrm{t}) \quad \text { and } \quad \widetilde{\delta}:=(\mathrm{q}-1) \mathrm{t} \delta(\mathrm{t}) \text { are independent of } \mathrm{t} \in \mathbb{T} \text {, }
$$

then Proposition 4.4 gives us that (20) is satisfied and that the Cobb-Douglas production function is defined and equals

$$
f(x)=\frac{x}{\widetilde{s}}\left\{\widetilde{\delta}-1+\left(\frac{1+(1-\alpha) \widetilde{s} x^{\alpha-1}}{1+(1-\alpha) \widetilde{\delta}}\right)^{\frac{1}{1-\alpha}}\right\} .
$$

Using (21), we rewrite equation (18) in the form (8), i.e., as a Bernoulli equation on time scales.

Theorem 4.8. Assume (10) and (20) and let $\mathrm{f}$ be defined by (21). Then (18) holds if and only if

$$
k^{\Delta}(t)=\left\{w \ominus\left(\frac{1}{\alpha-1} \odot\left(g k^{\alpha-1}\right)\right)\right\}(t) k(t) .
$$


Example 4.9. If $\mathbb{T}=\mathbb{R}$, then $w=-\delta$, and equation (26) is

$$
k^{\prime}(t)=\left(s k^{\alpha-1}(t)-\delta\right) k(t) .
$$

Hence equation (26) is indeed a generalized form of the continuous Solow model with the CobbDouglas production function.

With the generalized Cobb-Douglas function, we can find the solution of the Solow model (18) on time scales.

Theorem 4.10. Assume (10) and

$$
\mathrm{K}:=\frac{\mathrm{s}(\mathrm{t})}{\delta(\mathrm{t})} \quad \text { is independent of } \mathrm{t} \in \mathbb{T}
$$

and define $\mathrm{p} \in \mathcal{R}$ by

$$
p(t):=(1-\alpha) \delta(t) \quad \text { for all } \quad t \in \mathbb{T} .
$$

Then the solution of (26) with initial condition $\mathrm{k}\left(\mathrm{t}_{0}\right)=\mathrm{k}_{0}>0$, where $\mathrm{t}_{0} \in \mathbb{T}$, is given by

$$
k(t)=\left\{k+\frac{k_{0}^{1-\alpha}-\kappa}{e_{p}\left(t, t_{0}\right)}\right\}^{\frac{1}{1-\alpha}} \quad \text { for all } t \in \mathbb{T},
$$

provided the quantity in curly braces in (29) is always positive.

Proof. Suppose k solves (26) such that $k\left(t_{0}\right)=k_{0}$. Define $\widetilde{x}:=k^{\alpha-1}$. By [8, Theorem 2.37], we have

$$
\begin{aligned}
\frac{\tilde{x}^{\Delta}}{\widetilde{x}} & =(\alpha-1) \odot \frac{k^{\Delta}}{k} \\
& =(\alpha-1) \odot\left\{w \ominus\left[\frac{1}{\alpha-1} \odot\left(g k^{\alpha-1}\right)\right]\right\} \\
& =[(\alpha-1) \odot w] \ominus\left(g k^{\alpha-1}\right) \\
& =(\delta(1-\alpha)) \ominus\left(g k^{\alpha-1}\right)
\end{aligned}
$$

SO

$$
\widetilde{x}^{\Delta}=(p \ominus(g \tilde{x})) \widetilde{x},
$$

which shows that $\tilde{x}$ solves the logistic equation on time scales (see [1] and [8, Section 2.4]). Define $y:=1 / \widetilde{x}$. Then

$$
y^{\Delta}=\left(\frac{1}{\tilde{x}}\right)^{\Delta}=\frac{-\widetilde{x}^{\Delta}}{\widetilde{x} \widetilde{x}^{\sigma}}=-(p \ominus(g \widetilde{x})) y^{\sigma}=\frac{g \widetilde{x}-p}{1+\mu g \widetilde{x}} y^{\sigma}
$$

and hence

$$
(1+\mu g \widetilde{x}) y^{\Delta}=g \widetilde{x} y^{\sigma}-p y^{\sigma},
$$

i.e., using the "simple useful formula",

$$
y^{\Delta}+\left(y^{\sigma}-y\right) g \widetilde{x}=g \widetilde{x} y^{\sigma}-p y^{\sigma},
$$


i.e.,

$$
y^{\Delta}=-p y^{\sigma}+g
$$

Using

$$
g=(1-\alpha) s=(1-\alpha) \delta \kappa=\kappa p
$$

and the variation of constants formula [7, Theorem 2.74], the solution of (30) is given by

$$
\begin{aligned}
y(t) & =y_{0} e_{\ominus p}\left(t, t_{0}\right)+\int_{t_{0}}^{t} g(\tau) e_{\ominus p}(t, \tau) \Delta \tau \\
& =y_{0} e_{\ominus p}\left(t, t_{0}\right)+\int_{t_{0}}^{t} k p(\tau) e_{p}(\tau, t) \Delta \tau \\
& =y_{0} e_{\ominus p}\left(t, t_{0}\right)+k \int_{t_{0}}^{t} p(\tau) e_{p}(\tau, t) \Delta \tau \\
& =y_{0} e_{\ominus p}\left(t, t_{0}\right)+\left.k e_{p}(\tau, t)\right|_{t_{0}} ^{t} \\
& =y_{0} e_{\ominus p}\left(t, t_{0}\right)+k\left(1-e_{\ominus p}\left(t, t_{0}\right)\right) .
\end{aligned}
$$

From the substitutions we performed, we have that $y_{0}=k_{0}^{1-\alpha}$ as well as $k(t)=\frac{1}{y(t)^{\frac{1}{\alpha-1}}}$, which shows (29). Conversely, $k$ given by (29) is easily seen to be a solution of (26).

Using Theorem 4.10, we obtain the asymptotic stability of the unique equilibrium point of (26).

Theorem 4.11. Assume (10) and (27). If

$$
\int_{t_{0}}^{\infty} \delta(t) \Delta t=\infty
$$

then any solution $\mathrm{k}$ of (26) in the form (29) satisfies

$$
\lim _{t \rightarrow \infty} k(t)=k^{\frac{1}{1-\alpha}}=: \bar{k},
$$

and $\overline{\mathrm{K}}$ is the unique equilibrium point of (26).

Proof. We have $p(t)>0$ for all $t \in \mathbb{T}$, where $p$ is defined in Theorem 4.10. Hence $p \in \mathcal{R}^{+}$. Thus, by [4, Remark 2], we have

$$
e_{p}\left(t, t_{0}\right) \geq 1+\int_{t_{0}}^{t} p(\tau) \Delta \tau=1+(1-\alpha) \int_{t_{0}}^{t} \delta(\tau) \Delta \tau \text { for all } t \geq t_{0}
$$

Therefore, using (31),

$$
\lim _{t \rightarrow \infty} e_{p}\left(t, t_{0}\right)=\infty
$$

and thus

$$
\lim _{t \rightarrow \infty} k(t)=\left\{k+\frac{k_{0}^{1-\alpha}-k}{\lim _{t \rightarrow \infty} e_{p}\left(t, t_{0}\right)}\right\}^{\frac{1}{1-\alpha}}=k^{\frac{1}{1-\alpha}}=\bar{k}
$$


Now we show that $\bar{\kappa}$ is the unique nontrivial equilibrium point of (26): A point $\underline{\underline{K}}$ is a nontrivial equilibrium point of (26) if and only if

$$
w \ominus\left(\frac{1}{\alpha-1} \odot\left(\mathrm{gk}^{\alpha-1}\right)\right)=0,
$$

which holds if and only if (use the definition of $w$, (27), and the properties of $\ominus$ )

$$
\frac{1}{\alpha-1} \odot \frac{g}{\kappa}=\frac{1}{\alpha-1} \odot\left(\underline{g}^{\alpha-1}\right),
$$

which is true if and only if (use the properties of $\odot$ and the definition of $g$ )

$$
\frac{1}{\kappa}=\underline{\kappa}^{\alpha-1},
$$

i.e., $\underline{\kappa}=\bar{\kappa}$. This completes the proof.

Example 4.12. Let $\mathbb{T}=\mathbb{R}$ and assume (10) and (27). Then (29) reads

$$
k(t)=\left\{k+\frac{k_{0}^{1-\alpha}-k}{e^{(1-\alpha) \int_{t_{0}}^{t} \delta(\tau) \Delta \tau}}\right\}^{\frac{1}{1-\alpha}} .
$$

If, in addition, $\mathrm{t}_{0}=0$ and $\delta$ is constant (this implies that $\mathrm{s}$ is constant as well), then

$$
k(t)=\left\{k+\frac{k_{0}^{1-\alpha}-k}{e^{(1-\alpha) \delta t}}\right\}^{\frac{1}{1-\alpha}} .
$$

Example 4.13. Let $\mathbb{T}=\mathrm{h} \mathbb{Z}$ with $\mathrm{h}>0$ and assume (10) and (27). Then (29) reads

$$
k(t)=\left\{k+\frac{k_{0}^{1-\alpha}-k}{\prod_{i=t_{0} / h}^{t / h-1}(1+(1-\alpha) h \delta(i h))}\right\}^{\frac{1}{1-\alpha}} .
$$

If, in addition, $\mathrm{t}_{0}=0$ and $\delta$ is constant (this implies that $\mathrm{s}$ is constant as well), then

$$
k(t)=\left\{k+\frac{k_{0}^{1-\alpha}-\kappa}{(1+(1-\alpha) h \delta)^{t / h}}\right\}^{\frac{1}{1-\alpha}} .
$$

Example 4.14. Let $\mathbb{T}=\mathrm{q}^{\mathbb{N}_{0}}$ with $\mathrm{q}>1$ and assume (10) and (27). Then (29) reads

$$
k(t)=\left\{k+\frac{k_{0}^{1-\alpha}-k}{\prod_{i=\log _{q} t_{0}}^{\log _{q} t-1}\left(1+(q-1) q^{i}(1-\alpha) \delta\left(q^{i}\right)\right)}\right\}^{\frac{1}{1-\alpha}} .
$$


If, in addition, $\mathrm{t}_{0}=1$ and $\widetilde{\delta}:=(\mathrm{q}-1) \mathrm{t} \delta(\mathrm{t})$ is constant (this implies that $(\mathrm{q}-1) \mathrm{ts}(\mathrm{t})$ is constant as well), then

$$
k(t)=\left\{k+\frac{k_{0}^{1-\alpha}-k}{(1+(1-\alpha) \widetilde{\delta})^{\log _{q} t}}\right\}^{\frac{1}{1-\alpha}} .
$$

\section{Properties of the Production Function}

In this section, we show that our Cobb-Douglas production function $f$ given in (21) satisfies the time scales Inada conditions (see [2, 3])

$$
\left\{\begin{array}{c}
f(x)>0, \quad \tilde{f}^{\prime}(x)<0, \quad f^{\prime \prime}(x)<0 \quad \text { for all } x>0, \\
\widetilde{f}(x)>\zeta(t) \geq 0, \quad f^{\prime}(x)>\zeta(t) \geq 0 \quad \text { for all } \quad x>0 \text { and all } t \in \mathbb{T}, \\
\lim _{x \rightarrow 0^{+}} \tilde{f}(x)=\lim _{x \rightarrow 0^{+}} f^{\prime}(x)=\infty, \\
\lim _{x \rightarrow \infty} \widetilde{f}(x)=\lim _{x \rightarrow \infty} f^{\prime}(x)=\zeta(t) \geq 0 \quad \text { for all } t \in \mathbb{T},
\end{array}\right.
$$

where $\zeta: \mathbb{T} \rightarrow \mathbb{R}$ is defined in the following lemma.

Lemma 5.1. Assume (10) and define

$$
\zeta(t):=\frac{1}{s(t)}\left\{\delta(t)+\left(\frac{1}{\alpha-1} \odot((1-\alpha) \delta)\right)(t)\right\} .
$$

Then $\zeta(\mathrm{t}) \geq 0$.

Proof. If $\mu(t)=0$, then $\zeta(t)=0$. If $\mu(t)>0$, then, as in the proof of Proposition 4.3, we have

$$
\left(\frac{1}{\alpha-1} \odot((1-\alpha) \delta)\right)(t)=\frac{(1+(1-\alpha) \mu(t) \delta(t))^{\frac{1}{\alpha-1}}-1}{\mu(t)} .
$$

Now using the well-known Bernoulli inequality, we obtain

$$
(1+(1-\alpha) \mu(t) \delta(t))^{\frac{1}{\alpha-1}} \geq 1+\frac{1}{\alpha-1}(1-\alpha) \mu(t) \delta(t)=1-\mu(t) \delta(t) .
$$

This proves the claim.

Theorem 5.2. Assume (10) and (20) and define the Cobb-Douglas production function by (21). If there exists $\mathrm{t} \in \mathbb{T}$ such that $\mu(\mathrm{t})=0$, then the Cobb-Douglas production function satisfies the Inada conditions (32).

Proof. By Theorem 4.1 and (20),

$$
f(x)=x^{\alpha} \text { and } \tilde{f}(x)=x^{\alpha-1} .
$$

Clearly, $f$ given by (33) satisfies the Inada conditions (32). 
Theorem 5.3. Assume (10) and (20) and define the Cobb-Douglas production function by (21). If there exists $\mathrm{t} \in \mathbb{T}$ such that $\mu(\mathrm{t})>0$, then the Cobb-Douglas production function satisfies the Inada conditions (32).

Proof. By Theorem 4.3 and (20), we have

$$
\widetilde{f}(x)=\frac{1}{\mu(t) s(t)}\left\{\mu(t) \delta(t)-1+\left(\frac{1+(1-\alpha) \mu(t) s(t) x^{\alpha-1}}{1+(1-\alpha) \mu(t) \delta(t)}\right)^{\frac{1}{1-\alpha}}\right\} .
$$

In order to check that the Inada conditions (32) are satisfied, we use the following notation:

$$
\left\{\begin{array}{c}
\widetilde{\delta}:=\mu(t) \delta(t), \quad \kappa:=\frac{s(t)}{\delta(t)}, \quad \widetilde{\alpha}:=(1-\alpha) \widetilde{\delta} \\
\zeta:=\frac{1}{\widetilde{\delta} \kappa}\left\{\widetilde{\delta}-1+(1+\widetilde{\alpha})^{\frac{1}{\alpha-1}}\right\}, \quad z(x):=\frac{1+\widetilde{\alpha} \kappa \chi^{\alpha-1}}{1+\widetilde{\alpha}} .
\end{array}\right.
$$

Using (35), we rewrite (34) as

$$
\widetilde{f}(x)=\frac{1}{\widetilde{\delta} K}\left\{\widetilde{\delta}-1+(z(x))^{\frac{1}{1-\alpha}}\right\} .
$$

We obviously have

$$
z(x)>\frac{1}{1+\widetilde{\alpha}} \text { for all } x>0 .
$$

Using (37) in (36), we find

$$
\widetilde{f}(x)>\frac{1}{\widetilde{\delta}_{K}}\left\{\widetilde{\delta}-1+(1+\widetilde{\alpha})^{\frac{1}{\alpha-1}}\right\}=\zeta \text { for all } x>0 .
$$

By Lemma 5.1, we also have $\zeta \geq 0$, and hence $\widetilde{f}(x)>0$ for all $x>0$ so that $f(x)=x \widetilde{f}(x)>0$ for all $x>0$. Next, note that

$$
z^{\prime}(x)=\frac{\widetilde{\alpha}(\alpha-1) \kappa}{1+\widetilde{\alpha}} x^{\alpha-2} .
$$

Using (38) in (36), we find

$$
\begin{aligned}
\widetilde{f}^{\prime}(x) & =\frac{1}{\widetilde{\delta} \kappa(1-\alpha)}(z(x))^{\frac{1}{1-\alpha}-1} z^{\prime}(x) \\
& =\frac{1}{\widetilde{\delta} \kappa(1-\alpha)}(z(x))^{\frac{\alpha}{1-\alpha}} \frac{\widetilde{\alpha}(\alpha-1) \kappa x^{\alpha-2}}{1+\widetilde{\alpha}} \\
& =-\frac{\widetilde{\alpha} x^{\alpha-2}}{(1+\widetilde{\alpha}) \widetilde{\delta}}(z(x))^{\frac{\alpha}{1-\alpha}}<0 \text { for all } x>0 .
\end{aligned}
$$


Using this, (36), (35), (37), and the product rule for $f(x)=x \widetilde{f}(x)$, we get

$$
\begin{aligned}
f^{\prime}(x) & =\widetilde{\mathbf{f}}(x)+x \widetilde{f}^{\prime}(x) \\
& =\frac{1}{\widetilde{\delta} K}\left\{\widetilde{\delta}-1+(z(x))^{\frac{1}{1-\alpha}}\right\}-\frac{\widetilde{\alpha} x^{\alpha-1} \kappa}{(1+\widetilde{\alpha}) \widetilde{\delta} K}(z(x))^{\frac{\alpha}{1-\alpha}} \\
& =\frac{1}{\widetilde{\delta} K}\left\{\widetilde{\delta}-1+(z(x))^{\frac{1}{1-\alpha}}\right\}-\left(z(x)-\frac{1}{1+\widetilde{\alpha}}\right) \frac{1}{\widetilde{\delta}_{K}}(z(x))^{\frac{\alpha}{1-\alpha}} \\
& =\frac{1}{\widetilde{\delta} K}\left\{\widetilde{\delta}-1+\frac{1}{1+\widetilde{\alpha}}(z(x))^{\frac{\alpha}{1-\alpha}}\right\} \\
& >\frac{1}{\widetilde{\delta} K}\left\{\widetilde{\delta}-1+\frac{1}{1+\widetilde{\alpha}}(1+\widetilde{\alpha})^{\frac{\alpha}{\alpha-1}}\right\} \\
& =\frac{1}{\widetilde{\delta} K}\left\{\widetilde{\delta}-1+(1+\widetilde{\alpha})^{\frac{1}{\alpha-1}}\right\}=\zeta,
\end{aligned}
$$

i.e.,

$$
f^{\prime}(x)=\frac{1}{\widetilde{\delta}_{K}}\left\{\widetilde{\delta}-1+\frac{1}{1+\widetilde{\alpha}}(z(x))^{\frac{\alpha}{1-\alpha}}\right\}>\zeta \text { for all } x>0
$$

Also, since

$$
\lim _{x \rightarrow 0^{+}} z(x)=\infty \quad \text { and } \quad \lim _{x \rightarrow \infty} z(x)=\frac{1}{1+\widetilde{\alpha}},
$$

we find from (36) and (39) that

$$
\lim _{x \rightarrow 0^{+}} \tilde{f}(x)=\lim _{x \rightarrow 0^{+}} f^{\prime}(x)=\infty
$$

and

$$
\lim _{x \rightarrow \infty} \widetilde{f}(x)=\lim _{x \rightarrow \infty} f^{\prime}(x)=\zeta \geq 0 .
$$

Finally, using (39) and (38), we obtain

$$
\begin{aligned}
f^{\prime \prime}(x) & =\frac{1}{\widetilde{\delta} k(1+\widetilde{\alpha})} \frac{\alpha}{1-\alpha}(z(x))^{\frac{\alpha}{1-\alpha}-1} z^{\prime}(x) \\
& =\frac{1}{\widetilde{\delta} \kappa(1+\widetilde{\alpha})} \frac{\alpha}{1-\alpha} \frac{\widetilde{\alpha}(\alpha-1) \kappa}{1+\widetilde{\alpha}} \chi^{\alpha-2}(z(x))^{\frac{2 \alpha-1}{1-\alpha}} \\
& =-\frac{\alpha \widetilde{\alpha}}{\widetilde{\delta}(1+\widetilde{\alpha})^{2}} x^{\alpha-2}(z(x))^{\frac{2 \alpha-1}{1-\alpha}}<0 \text { for all } x>0 .
\end{aligned}
$$

This shows that all conditions in (32) are satisfied.

\section{General Solow Model on Time Scales}

Let us now assume (10) and (11) so that we allow now that the technology develops exponentially and/or the population increases exponentially. All results from Section 4 and Section 5 still hold true when we replace $s$ and $\delta$ in (18) by

$$
\frac{s}{1+\mu(n \oplus r)} \quad \text { and } \quad \frac{\delta+(n \oplus r)}{1+\mu(n \oplus r)}
$$


respectively, as (18) in this case results in (15). This means that $w$ and $g$ from (19) are replaced by

$$
w(t)=\left(\frac{1}{\alpha-1} \odot \frac{(\delta+(n \oplus r)) g}{s}\right)(t) \quad \text { and } \quad g(t)=\frac{(1-\alpha) s(t)}{1+\mu(t)(n \oplus r)(t)},
$$

respectively. Next, $\widetilde{f}(x)$ in (20) is replaced by

$$
\frac{\delta(t)+(n \oplus r)(t)+(1+\mu(t)(n \oplus r)(t))\left(w \ominus\left(\frac{1}{\alpha-1} \odot\left(g x^{\alpha-1}\right)\right)\right)}{s(t)} .
$$

Moreover, $k$ and $p$ in (27) and (28) are replaced by

$$
\frac{s(t)}{\delta(t)+(n \oplus r)(t)} \quad \text { and } \quad(1-\alpha) \frac{\delta(t)+(n \oplus r)(t)}{1+\mu(t)(n \oplus r)(t)},
$$

respectively. Finally, condition (31) turns into

$$
\int_{t_{0}}^{\infty} \frac{\delta(t)+(n \oplus r)(t)}{1+\mu(t)(n \oplus r)(t)} \Delta t=\infty
$$

Received: January 2013. Revised: February 2013.

\section{References}

[1] E. Akin-Bohner and M. Bohner. Miscellaneous dynamic equations. Methods Appl. Anal., 10(1):11-30, 2003.

[2] P. Barelli and S. de Abreu Pessôa. Inada conditions imply that production function must be asymptotically Cobb-Douglas. Econom. Lett., 81(3):361-363, 2003.

[3] G. Beer. The Cobb-Douglas Production Function. Math. Mag., 53(1):44-48, 1980.

[4] M. Bohner. Some oscillation criteria for first order delay dynamic equations. Far East J. Appl. Math., 18(3):289-304, 2005.

[5] M. Bohner, G. Gelles, and J. Heim. Multiplier-accelerator models on time scales. Int. J. Stat. Econ., 4(S10):1-12, 2010.

[6] M. Bohner and G. M. Gelles. Risk aversion and risk vulnerability in the continuous and discrete case. A unified treatment with extensions. Decis. Econ. Finance, 35:1-28, 2012.

[7] M. Bohner and A. Peterson. Dynamic equations on time scales. Birkhäuser Boston Inc., Boston, MA, 2001. An introduction with applications.

[8] M. Bohner and A. Peterson, editors. Advances in dynamic equations on time scales. Birkhäuser Boston Inc., Boston, MA, 2003. 
[9] G. Gandolfo. Economic dynamics. North-Holland Publishing Co., 1997.

[10] S. Hilger. Ein Maßkettenkalkül mit Anwendung auf Zentrumsmannigfaltigkeiten. PhD thesis, Universität Würzburg, 1988.

[11] L. Ljungqvist and T. J. Sargent. Recursive Macroeconomic Theory. MIT Press, 2000.

[12] R. M. Solow. A contribution to the theory of economic growth. The Quarterly Journal of Economics, 70(1):65-94, February 1956.

[13] Swan. Economic growth and capital accumulation. Economic Record, 32:334-361, 1956.

[14] C. C. Tisdell and A. Zaidi. Basic qualitative and quantitative results for solutions to nonlinear, dynamic equations on time scales with an application to economic modelling. Nonlinear Anal., 68(11):3504-3524, 2008. 